\title{
Analysis on the discrimination of synonyms
}

\author{
Qing Luo \\ Nanchang institute of science \& technology, China
}

Keywords: synonym, difference, discrimination

\begin{abstract}
The research of synonyms in modern Chinese language has always been a seemingly simple problem, but actually quite difficult problem. This paper will combine with examples, to analyze the differences between synonyms, in order to better guide people to correctly understand and use the motherland language, to promote the standardization of language.
\end{abstract}

\section{The nature of the synonyms}

People mainly based on the similarities and differences of concept to determine whether the words are synonymous before 1960s, this perspective is directly derived from the former Soviet Union scholar. What is the meaning of "similarities" of synonyms? Some scholars think that is the same concepts, some scholars think that "reflected objects are same" or "referred objects are same".

The differences caused by the term of "synonyms", mainly aimed at such questions: the "similarities" of the "synonyms" are same, or similar? The synonyms are equivalents or near-synonyms? In summary, there are three kinds of formulations:

(1) The statement of "meaning is same or close"

The 《Example solution of synonyms》 of Chuo Bo (The language learning, 1951, 2) first explicitly put forward:" Similar words in the sense of meanings are called synonyms". A lot of scholars hold this view, such as Mingkai Gao (1955) thinks that "Synonyms are the words with similar meanings", Shilu Zhang (1956) thinks that "As long as the meanings are close, also can belong to synonyms". Some scholars hold different opinions, completely contrary to the above statement of "meanings are same or close". Li Wang (1953) thinks that: "Synonyms are two words or more words with the same meanings". But more and more scholars advocate that synonyms including two types, one is the words with complete and equal meanings, another is the words with not complete and equal meanings. Such as Zumo Zhou(1956) points out that "Synonyms are the words with the same meanings or very similar meanings".

(2) The statement of " concept is same"

This kind of view thinks that synonyms are the words with a variety of subtle differences within the same concept. The view of "Different synonyms express the nuances of the concept in different way" is accepted by many scholars in the field of linguistics in China. Such as: Fuyuan Cui (1957) thinks that "Synonyms are the words with a variety of subtle differences within the same concept".

(3) The statement of "object is same"

Different from the above situation, some scholars from the objects reflected by the meaning of words whether are consistent to talk about the problems of synonyms and non synonyms. Such as Changxu Sun (1956) points out that "The only one basis to distinguish synonyms is whether they generalize the same object". The responders are not many within 20 years, until after the middle of 70s, Xingjian Li and Shuxin Liu (1975) begin to agree with this view, they think that "The meanings of two words are not far, but don't reflect the same thing, there is no synonymous relationship, they are near-synonyms, not are synonyms". Shuxin Liu (1987) further plays to the 
point of view, and puts it as the only basis to distinguish synonyms and near synonyms: "Two words no matter how the differences in meanings, if they refer to the same object, they must constitute synonyms. On the other hand, although the meanings of two words are very close, if they do not refer to the same object, then they are just near synonyms, can’t be regarded as synonyms".

\section{The composition of synonyms}

(1) From the point of the voice

A. Voice is completely different. The voice of synonyms is completely different refers to two words or a few words have no same place in syllable form, such as "pretty", "beautiful" and "good looking", their phonetic form is respectively no common form of syllables.

B. Voice is not exactly the same. The voice of synonyms is not exactly the same refers to two words or a few words in syllable form have both same and different, such as "nature" and "essence", their phonetic form on the syllables has same and different, voice is not exactly the same. The voice of synonyms is not exactly the same, word formation morpheme is entirely different or is not exactly the same is the main body of synonyms, it is the focus for us to research the synonyms.

C. Constituted by same morphemes, but the order is different. A set of synonyms constituted by the same morphemes, reversed the order, reversed the construction mode of behind two words maybe is same, maybe is different. For example: "envy" and "jealousy", they are a set of synonyms, both are constituted by two morphemes of "envy" and "jealous", the construction mode of these two words all are joint type, but order of morphemes is different.

\section{The discrimination of synonyms}

Learning synonyms should not only master the similarities, but also grasp the differences. By comparison: "similarities" are easy to find, but "differences" are hard to find. Therefore, the focus to discriminate the synonyms should be placed on the "differences".

(1) The theory and methods of synonyms discrimination

The theory of synonyms analysis mainly refers to the two aspects of word meaning contents theory and word meaning characteristics theory. The content of word meaning is summed up in three parts, including lexical meaning, grammatical meaning and color meaning, lexical meaning is most important in these three word meanings. True synonyms from the point of word meaning characteristics, includes the objectivity characteristics of word meaning, general characteristics of word meaning, social characteristics of word meaning, subjective characteristics of word meaning, developmental characteristics of word meaning, nationality characteristic of word meaning, concept correspondence characteristic of word meaning, specific things correspondence characteristic of word meaning and so on. Among these characteristics, behind two characteristics have important influence on the synonyms. Characteristic theory of word meaning is the theory support of synonyms research and synonyms analysis, there is another kind of situation is that the two words both are made up of two identical syllables form, but the order of the syllables is different, that is the so-called "contrary-order synonyms".

(2) From the point of the constituted morpheme

A. Word formation morpheme is totally different or not completely same. The word formation morpheme of synonyms is totally different or not completely same is the main body of synonyms, is the focus for us to research synonyms.

B. Constituted by same morphemes, but the order is different. A set of synonyms constituted by the same morphemes, reversed the order, reversed the construction mode of behind two words 
maybe is same, maybe is different.

(3) The discrimination of synonyms

Learning synonyms should not only master the similarities, but also grasp the differences. By comparison: "similarities" are easy to find, but "differences" are hard to find. Therefore, the focus to discriminate the synonyms should be placed on the "differences".

A. The theory and methods of synonym discrimination

The theory of synonyms analysis mainly refers to the two aspects of word meaning contents theory and word meaning characteristics theory. the content of word meaning is summed up in three parts, including lexical meaning, grammatical meaning and color meaning, lexical meaning is most important in these three words meanings. True synonyms from the point of word meaning characteristics, includes the objectivity characteristics of word meaning, general characteristics of word meaning, social characteristics of word meaning, subjective characteristics of word meaning, developmental characteristics of word meaning, nationality characteristic of word meaning, concept correspondence characteristic of word meaning, specific things correspondence characteristic of word meaning and so on. Among these characteristics, behind two characteristics have important influence on the synonyms. Characteristic theory of word meaning is the theory support of synonyms research and synonyms analysis, it has a positive guiding significance to the synonyms research.

B. The steps and methods of the synonyms discrimination

The analysis of synonyms should include the following steps:

The first step is to determine the analysis of a set of words, the second step is to conclude the similarities, the third step is to gather materials, the fourth step is to find out differences through analysis, these differences include the following contents: (1)the collocation requirements on the voice, (2) the differences of significance, including the difference of color meaning and the difference of semantic category detail classify, (3) the situation of acting as parts of the sentence on grammar, the position presented in sentence, choice of sentence patterns, etc., (4) the difference of pragmatic habit collocation and commonly used (word frequency), etc., the last step is to summarize, explain the similarities and differences of synonyms in items.

C. The method of synonyms discrimination

When discriminate the synonyms, analyze the differences of synonyms is one of the most important contents to analyze the synonyms. The "similarities" and "differences" of synonyms in pragmatics both are the contents need to focused for synonyms analysis. Here are a few aspects to talk about as follows:

a. From the aspects of word meaning to discriminate.(1) The weight of the word meaning is different. The nuances of some synonyms refers to the weight of the mood is different. Such as a set of synonyms for "damage" and "destroy", both have the meaning of "make it damaged", but the weight of mood is different. "Damage" means the degree of the damage is lighter, usually is unintentional, word meaning is lighter. "Destroy" must be intentional, the degree of the damage is heavier. (2) The range and size is different. Although some synonyms reflect the same object, but refer to the range and size is different. For example: "nature" and "quality" all express the properties, but "nature" can refer to the properties of everything, "quality" generally refers to the characteristics of person's behavior and spiritual cultivation, meaning scope is smaller than "nature".(3)Objects are different. The concept of some synonyms represented is the same, but applicable objects are different, there are the difference of up and down, inside and outside, etc.

b. From the aspects of color to discriminate. Synonyms in color sense also exist a problem of coordination, the members of different stylistic coloring and emotion coloring are greatly affected 
the use of synonyms. (1) Stylistic coloring. Among the synonyms, some words are suitable for writing or solemn occasions, with a solemn style coloring. Some words are suitable for oral or general occasions, with a popular language coloring. (2) Emotional coloring. The basic meaning of some words are the same, just emotional coloring is different. Some words with the meaning of love, respect and praise, called commendatory word, such as "decisive". Some words with the meaning of loathing and ironic, called derogatory term, such as "arbitrary". There also have some words without the meaning of praise or blame, that is the neutral words, such as "overturn".

c. From the aspects of collocation of words is different to discriminate. The basic meaning of some synonyms is the same, but the collocation is different, can't mixed to use.

d. From the aspects of grammar to discriminate. The synonyms on the grammar function are the same, grammar function is same mainly refers to the ability to act as parts of the sentence is the same, a lot of synonyms on the combination of the grammar have no difference, but some other performance aspects of synonyms in grammar is still not completely equivalent, such as location situation appears in the sentence, the choice of sentence patterns, semantic category detail classify, specific pragmatic collocation, etc., these aspects may exist a certain differences.

\section{Conclusion}

This paper summarizes and analyzes the definition of synonyms in the field of modern Chinese for senior scholars, and analyzes synonyms from three aspects: the nature of the synonyms, the composition of synonyms and the discrimination of synonyms, but still have some questions need to be researched. 1. The indeed problem for the name and the connotation of synonyms and near-synonyms. Some people thought that synonyms should contain near-synonyms, some people thought that their name is different, their connotation is also different. 2. When constitute a set of synonyms, whether require the part of speech is the same, also exist some differences. Therefore, this paper doesn't discuss this problem.

\section{References}

[1] Yongfeng Luo. Some thoughts on modern Chinese synonyms. [J]. Journal of Jiangxi Institute of Education (Social Science), 1999, 5.

[2] Xiangdong Xing. Several questions about determining the scope of the synonyms. [J]. Journal of Language and Literature, 1985, (2).

[3] Weihan Xu. Chinese vocabulary study in twentieth Century. [M]. Taiyuan: Sea of Books Press, 2000.

[4] Huaiqing Fu. Modern Chinese vocabulary (an enlarged revision). [M]. Beijing: Peking University Press, 2004.

[5] Zhimin Xu. Commendatory words and derogatory words can be composed of synonyms? [J]. Chinese Teaching Communication, 1980, (2).

[6] Bin Zhang. New modern Chinese. [M]. Shanghai: Fudan University Press, 2002. 